Final Report on the DOE Grant

DOE DEFG 02-97ER13939

\title{
PERFORMANCE-DRIVEN ROBUST \\ IDENTIFICATION AND CONTROL OF \\ UNCERTAIN DYNAMICAL SYSTEMS
}

TAMER BAŞAR

Principal Investigator

Decision and Control Laboratory

Coordinated Science Laboratory

University of Illinois at Urbana-Champaign

1308 West Main Street

Urbana, Illinois 61801

Submitted to

U.S. Department of Energy

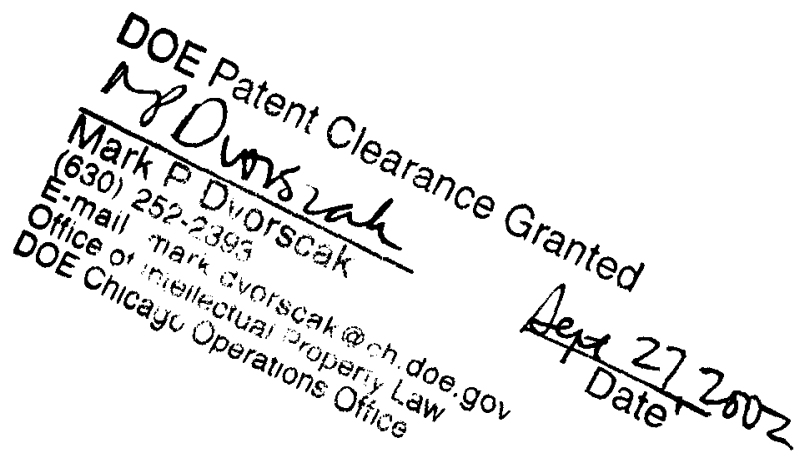

Office of Basic Energy Research

Division of Engineering and Geosciences

Attn: Dr. Robert Price

Period covered: June 15, 1997 - June 14, 2001

October 29, 2001 


\section{DISCLAIMER}

This report was prepared as an account of work sponsored by an agency of the United States Government. Neither the United States Government nor any agency Thereof, nor any of their employees, makes any warranty, express or implied, or assumes any legal liability or responsibility for the accuracy, completeness, or usefulness of any information, apparatus, product, or process disclosed, or represents that its use would not infringe privately owned rights. Reference herein to any specific commercial product, process, or service by trade name, trademark, manufacturer, or otherwise does not necessarily constitute or imply its endorsement, recommendation, or favoring by the United States Government or any agency thereof. The views and opinions of authors expressed herein do not necessarily state or reflect those of the United States Government or any agency thereof. 


\section{DISCLAIMER}

Portions of this document may be illegible in electronic image products. Images are produced from the best available original document. 


\title{
FINAL REPORT
}

on the DOE Grant

DOE DEFG 02-97ER13939

\section{Performance-Driven Robust Identification and Control of Uncertain Dynamical Systems}

\author{
by
}

TAMER BAŞAR

\section{INTRODUCTION}

The grant DEFG 02-97ER13939 from the Department of Energy has supported our research program on robust identification and control of uncertain dynamical systems, initially for the three-year period June 15, 1997-June 14, 2000, which was then extended on a no-cost basis for another year until June 14, 2001. This final report provides an overview of our research conducted during this period, along with a complete list of publications supported by the Grant.

Within the scope of this project, we have studied fundamental issues that arise in modeling, identification, filtering, control, stabilization, control-based model reduction, decomposition and aggregation, and optimization of uncertain systems. The mathematical framework we have worked in has allowed the system dynamics to be only partially known (with the uncertainties being of both parametric or structural nature), and further the dynamics to be perturbed by unknown dynamic disturbances. Our research over these four years has generated a substantial body of new knowledge, and has led to new major developments in theory, applications, and computational algorithms. These have all been documented in various journal articles and book chapters, and have been presented at leading conferences, as to be described below.

A brief description of the results we have obtained within the scope of this project can be found in Section 3. To set the stage for the material of that section, we first provide in the next section (Section 2) a brief description of the issues that arise in the control of uncertain systems, and introduce several criteria under which optimality will lead to robustness and stability. Section 4 contains a list of references cited in these two sections. A list of our publications supported by the DOE Grant (covering the period June 15, 1997 - June 14, 2001) comprises Section 5 of the report.

Throughout the report, citations to references are given in two forms: Those that start with a letter (such as [BaBe95]) refer to those listed in Section 4, and those that start with "9" or "0" (such as [99-12], or [00-04]) refer to those listed in Section 5. 


\section{UNCERTAIN DYNAMICAL SYSTEMS AND PERFORMANCE-DRIVEN ROBUSTNESS}

\section{Uncertain dynamical systems and robustness}

In our research, we have viewed uncertain dynamical systems as collections of interconnected subsystems described by differential or difference equations, having two types of input-controlled and uncontrolled. Controlled inputs are determined by the designer, as functions of the on-line information available to each particular input. Uncontrolled inputs, on the other hand, are dynamic uncertainties that are external to the overall system, generated for example by the environment in which the system resides. We call this type of uncertainty dynamic, because it may change over time, with the term static uncertainty reserved for example for an unknown nonlinearity linking two subsystems. The dynamic uncertainty (which could also be called disturbance) may admit a complete stochastic description (i.e., it may be described precisely as a stochastic process), or it may be deterministic but completely unknown (possibly satisfying some known bounds), or it may admit a description in between these two - such as the case of a stochastic process with only partially known statistics. Another type of dynamic uncertainty would manifest itself as subsystems undergoing unexpected or even undetected structural changes, or as the physical links as well as informational links changing or breaking at random times. Such uncertainties are commonly modeled as piecewise-deterministic processes [BaHa84; $\mathrm{Da} 92]$, where the jumps from one discrete state to another (such as from the operational state of an existing link to its failure state, or from one operational mode to another), occur at random points in time, and are governed by some statistical laws - such as the case of continuous-time Markov chains [FlSo93; ElAgMo95]. Although occurring at random times and not being predictable, these jumps could nevertheless be observed either perfectly at the time when they occur or with some delay. Also, in some applications, the stochastic processes that determine these jumps would be partially controlled through the physical state of the system.

An important issue in the control of uncertain dynamical systems is the analysis and optimization of the degree of sensitivity of the overall performance attained by a control design to the presence of structured and unstructured uncertainty, unmodeled dynamics, and unmodeled static interconnections or failures [BaBe95; GrLi95]. We call a controller robust if, broadly speaking, under it the performance of the dynamic system is not affected much when the uncertainties and the unmodeled interconnections are "small," and the performance degradation is graceful when the uncertainty is moderate or even large [02-01]. Of course, the notion of robustness is not context-free, but is with respect to a given class of uncertainties and unmodeled subsystem interconnections or dynamics. It is also determined by an optimality criterion that provides a quantification of performance that is compatible with the goals of the designer. Introduction of such an optimality criterion, with an eye on robustness, also enables one to design controllers that exhibit "minimal sensitivity" to modeling inaccuracies and other types of uncertainty.

\section{Performance measures for uncertain systems}

Our approach to robust identification, filtering and control in connection with uncertain systems has been performance driven, which requires the introduction of an appropriate cost function (equivalently, performance index) or several cost functions quantifying different performance tradeoffs. Also, one has to adopt a criterion of optimality, consistent with the general objectives. There are several possibilities here, depending on the nature of the uncertainty. 
(a) Worst-case or minimax criterion. If the dynamic system has uncertainties with no probabilistic description, then one possible optimality criterion is to choose the controllers in such a way that the effect of the unknown disturbances on the output is suppressed to the extent possible. In mathematical terms this requirement can be rephrased as the minimization (over all controlled inputs) of the "gain" from the disturbances to the system outputs, where the gain can be defined as the maximum value of the ratio of an appropriate norm of the system output to a compatible norm of the disturbances. If the system is linear, and the output and the disturbances are restricted to Hilbert spaces of square-integrable functions, then the "gain" is the $\mathrm{H}^{\infty}$ norm of the system transfer function, leading to a class of problems known as $\mathrm{H}^{\infty}$-control, first formulated in the early 1980's [Za81], but solved completely only about a decade later [DoGlKhFr89; $\mathrm{Ba} 91 ; \mathrm{BaBe} 95 ; \mathrm{St} 92 ; \mathrm{GrLi} 95]$. Among the several alternative methods for solving the standard $\mathrm{H}^{\infty}$-control problem, the most versatile and effective one is the approach based on zero-sum dynamic/differential games [BaBe95; $\mathrm{BaO} 199]$. In this approach, the original $\mathrm{H}^{\infty}$-norm minimization problem is reduced to a simpler problem of finding a control (adapted to the given on-line information on the system state and the disturbance) that achieves the upper value of a two-person zero-sum dynamic/differential game where the controller is the minimizing player and the disturbance is the maximizing player. This dynamic game-theoretic approach, besides providing a simpler and more intuitive derivation for the solution of the standard $\mathrm{H}^{\infty}$-control problem, has led to the creation of new research topics and tractable problem formulations in nonlinear systems and control [BaBe95; Is93; IsAs92; IsKa95; JaBa95; Sc91; Sc92; DiBaBe93; PaBa95b], adaptive control [ChSp95; DiBa97; 97-01]; identification [DiPaBa95], distributed-parameter and infinite-dimensional systems [Bar94; 00-02], and systems with hybrid description of uncertainty [PaBa95a]. In each case the controller design problem of minimization of the "gain" from the disturbances to the outputs has been recast as a particular differential game endowed with information that is compatible with that of the controller of the original problem.

(b) Risk-sensitivity index. When the disturbances have a complete probabilistic description, which means that the underlying system is stochastic, the criterion to be used is the expectation of an appropriate cost (loss) function, which is to be minimized over all controllers adapted to the information field generated by the available (stochastic) measurements. If the loss function is taken as stage additive (for example, for continuous-time formulations, as an integral of time-localized cost), then the stochastic control problem is known as risk neutral. If, however, the loss function is an exponential of a total stage-additive one (i.e., exponential of an integral, in continuous time), then we have a risk-sensitive index -- which could characterize risk-averse (pessimistic) or risk-seeking (optimistic) situations, based on the sign of a parameter at the exponent of the exponential. The fact that such cost functions could lead to much different optimal controllers than the risk-neutral case has been long known for some special classes of systems [Ja73; BeSc85; Wh90], but the observation that such a risk function admits the interpretation of a worst case cost for a related problem with deterministic (unknown) disturbances of the type discussed in (a) above, which in some cases is an equivalence, is relatively new [FlMc92; GlDo88; JaBaEl94; RhSp91; UcFu89; Wh91; BaBe95; Na96; $\mathrm{PaBa} 96$; BeEl96]. This observation has led, in recent years, to a surge of interest on this class of problems, and especially the risk-averse case, as optimal controllers obtained under such a performance index have additional robustness properties attached to them. Our research on this topic during the term of this grant has brought new interpretations to robustness that is captured through a risk-sensitive formulation; details of this will be discussed in the next section.

(c) Hybrid descriptions. Hybrid system descriptions arise when the dynamic uncertainty is stochastic 
but with partially unknown statistics, and when the system has both deterministic and stochastic uncertainties. The optimality criterion relevant to such systems would be a combination of the "minimax criterion" and the "stochastic criterion" introduced above. One such scenario occurs when the system undergoes structural changes over time, induced either by control actions or by the environment, such as the case of a system switching from one structure (mode of operation) to another one (selected randomly from a finite set) at random times, according to a jump Markov process [BaHa84], while having also unknown deterministic disturbances in its dynamics. Our recent results on this class of models are described in some detail again in the next section.

(d) Receding-horizon optimality. Sometimes, the length of the horizon over which optimization has to be done is not known in advance, or even if it is known it may be deșirable (for reasons of computational complexity and/or implementation) to perform optimization on a much shorter interval, say of length $T$, and iteratively repeat this process by advancing the interval each time by an amount, say $\tau<T$. If $[t, t+T]$ is one such typical interval, one solves the optimization problem over this interval with an appropriately picked terminal cost at $t+T$, but implements the resulting controller on a much shorter interval, $[t, t+\tau]$. Then, at the next step, the interval of interest becomes $[t+\tau, t+\tau+T]$, and the same process is repeated. This method of construction of controllers by receding the horizon is a viable alternative to the regular global optimization-based design if the latter runs into major obstacles due to either computational or informational complexity.

(e) Decentralized decision making with multiple criteria. In a large scale system composed of several weakly interconnected subsystems, with each one controlled by a possibly different decision maker, the four categories of optimality criteria introduced above are applicable to individual subsystems as well as to the overall system. It is quite common and natural, however, that all information necessary for control purposes is not shared by all decision makers, and that they would not have the same local objectives. This leads to multi-criteria optimization problems with informational decentralization, to which the equilibrium solution concepts of dynamic game theory are applicable [BaOl99]. Among the relevant solution concepts here are the Nash and Stackelberg equilibria, with there various manifestations; some of our recent work here are [98-04; 99-04; 99-10; 99-11].

\section{MAJOR CONTRIBUTIONS}

During the past four years, we have addressed a large number of issues on the control of uncertain systems, spanning all five optimality measures discussed in the previous section. Among the issues considered are:

(i) Robust adaptive control of nonlinear systems in parametric-strict-feedback form;

(ii) Disturbance attenuating controllers for nonlinear systems with local optimality properties;

(iii) Robust control of underactuated systems;

(iv) Nonparametric approaches to identification and control under uncertainty;

(v) Optimal control of dynamic systems with a hybrid description of uncertainty;

(vi) Risk-sensitive designs for both filtering and control;

(vii) Robust controller design under a receding horizon criterion;

(viii) Viscosity solutions to Hamilton-Jacobi-Isaacs equations that arise in nonlinear $H^{\infty}$ control;

(ix) Robust control of infinite-dimensional systems; and

(x) Analysis and control of compressor systems for stability and performance. 
In each case we have made fundamental contributions to theory as well as to the development of computational algorithms, and in some cases we have made significant contributions to specific application domains. These contributions are discussed below, in this section, in seven categories.

The technical approach adopted was performance-based robustness analysis, with applications to problems that arise in parametric and nonparametric identification; regulation, tracking and adaptation for deterministic, stochastic and hybrid systems; and stabilization of dynamical systems under various types of uncertainty. Some of the mathematical and system-theoretic tools used are: cost-to-come function methods, the theory of dynamic games, singular and regular perturbations, deterministic and stochastic Lyapunov methods, artificial neural networks, generalized backstepping techniques, Markov chain decomposition and averaging methods, the center manifold theorem, and viscosity solutions.

\subsection{Robust adaptive control}

One of our goals in this research program has been to develop a comprehensive theory for controlling nonlinear uncertain systems utilizing least possible knowledge of the system dynamics. Toward this goal, we addressed in the early stages of this research (see [98-06]) the problem of output tracking for systems in strict feedback form, which exhibit two types of uncertainty: multiplicative parametric and additive continuous. Our approach is optimization-based, but this class of problems can also be viewed as (robust) adaptive control problems since in every effective controller design there is implicit learning of the true values of the unknown parameters even though the identification and controller design phases may not be totally decoupled. Under full-state measurements and a condition on persistency of excitation, [98-06] presents closed-form expressions for robust controllers with built-in learning capabilities, which attenuate the effect of the disturbances and lead to asymptotic output tracking of a desired reference signal. An important by-product of the analysis there is the finding that the robust adaptive controllers that meet the dual specifications of asymptotic tracking and disturbance attenuation are generally not certainty-equivalent, but are asymptotically so as the measure quantifying the designer's confidence in the parameter estimates goes to zero.

After completing [98-06], we worked on several important generalizations. One of these involved the output measurement case, with an additional (stable) zero dynamics, and without any assumption of persistency of excitation. The results obtained have been reported in [99-02], where we considered SISO nonlinear systems described in the parametric-output-feedback form and subject to bounded affine disturbance inputs with nonlinear output-dependent gains. We further took the system to be minimum phase with a known sign of the high-frequency gain, and the true parameters to lie in an arbitrary but known bounded convex set-both being natural assumptions with practical justification. To utilize the given a priori knowledge, we introduced soft projection on the parameter estimates to keep them uniformly bounded without requiring any persistency of excitation condition to be satisfied. The objective of the controller design was then to make the system output track a given reference trajectory and keep all closed-loop signals uniformly bounded such that a prespecified disturbance attenuation level is attained. We considered two different measurement schemes, with the available information to the controller progressively decreasing. Under the first scheme, the controller was allowed to use both the output and its derivative. The two-step design procedure adopted combined cost-to-come function based $\mathrm{H}^{\infty}$-filtering [ $\left.\mathrm{BaBe} 95\right]$ with the backstepping methodology to arrive at explicit expressions for the resulting optimal controllers. Subsequently we have removed the dependence of the control law on the derivative information by an alternate derivation of the filter equations and an ensuing singular perturbations analysis. An important unique feature of the resulting nonlinear controllers is that they are not certainty equivalent, since the covariance matrix associated with the parameter estimates is 
bounded away from zero to ensure bounded-input bounded-state stability. We should also mention that even though the design uses the given bounds on the parameters, it does not use any a priori information on either the initial states of the system or the norm of the disturbance. Another important feature of this design paradigm is that it provides an appropriate cost functional compatible with the given design specifications. In this regard, the development in this work has deviated from the common optimal control practice where the cost functional is fixed a priori. The controllers derived actually ensure a nonpositive upper value for a particular zero-sum two player differential game characterized by this cost functional. Furthermore they result in arbitrarily small levels of disturbance attenuation, at the expense of increased control effort.

These results have provided the setting on which we have build our more recent research on this general topic, as discussed below:

One of our focus points has been the class of nonlinear uncertain dynamic systems where the nonlinearities are not necessarily linearly parametrized (as in [98-06]), or are not parametrized at all and are completely unknown. The problem is again robust control for regulation or tracking, but this time in the absence of even structural information. One approach here, which we had proposed to investigate three years ago, is to use neural network models to identify (for control purposes) the unknown nonlinearities. What makes this an appealing approach is the fact that using appropriate neural network architectures, a large class of functions and their derivatives can be approximated on compact sets with arbitrary precision; see, for example, [PaSa93], [CaEu92] and [Po92]. In [98-02], we addressed in this framework the problem of robust identification for unknown nonlinear systems with additive disturbances, and obtained powerful results on asymptotic convergence. A subsequent challenge was to lift these results from the identification domain and extend them to the control domain, so as to apply to (say) tracking problems. Previous attempts to use neural network-based identifiers in controlling nonlinear systems had largely failed because of the negative effect of the approximation errors on the closed-loop system (such as leading to instability). We have resolved this problem in [99-07] for a class of disturbance-driven systems in strict-feedback form, where the unknown nonlinearities depend on the output which is to track a given reference signal. We derived two classes of controllers, one using full state and derivative information, and the other one only full state information, which guarantee the boundedness of all signals in the closed-loop system. Two other appealing features of these controllers are that, the tracking error can be driven to an arbitrarily small neighborhood of the origin arbitrarily fast (at the expense of increased control effort), and under some requirements on the reference signal (which guarantee persistency of excitation) adequate identification of the uncertain system can be achieved.

We have subsequently extended the results of [99-07] to nonlinear systems with more general types of nonlinearities, not necessarily dependent only on the output. More specifically, we have considered in [99-21] the class of single-input single-output nonlinear systems in strict-feedback form with additional stable zero dynamics, where the nonlinearities in the system dynamics are not known. The only assumption we make on these nonlinearities is that when they are approximated in terms of radial basis functions (using a particular neural-net architecture) the optimal parameters that characterize the approximation lie in a known compact set. Since the unknown nonlinearities in the system description are arbitrary, the approximation errors may not belong to $\mathcal{L}_{2}$; therefore, they are not viewed in our work as parts of the external disturbances whose effects are to be attenuated in the $\mathcal{L}_{2}$ sense. The question we address in the paper is that of designing a robust state-feedback controller under which the system output tracks a given signal arbitrarily well, and all signals in the closed-loop system remain bounded. The controller design procedure developed involves the use of the modified versions of the $\mathrm{H}^{\infty}$-filters presented in [98-06], the backstepping design methodology, and 
the use of radially unbounded functions. The resulting controllers achieve the output tracking of a reference signal arbitrarily well at the expense of increased control effort, as also demonstrated through simulation studies. Furthermore, the closed-loop signals satisfy a disturbance attenuation inequality with respect to an equivalent disturbance, comprising the external disturbances and the approximation errors. Moreover, if a relevant persistency of excitation condition is satisfied, the estimates of the parameters that characterize the neural net approximation of the unknown nonlinearities converge to some neighborhood of their optimal values.

After the completion of the two pieces of work summarized above, that is, [99-07] and [99-21], the next step was to accomplish all this without full-state information, with only output measurements available. Results on this important extension can be found in [99-22] and [01-02]. These can be viewed as generalizations of the results of [99-02] (discussed earlier in this subsection) to the case where the nonlinearities are not parametrized (but the uncertainty is structural), and those of [99-07] and [99-21] (described above) to the output feedback case. In [99-22], we assume that not only the output but also its derivative is available for feedback, with the case of only output measurement treated in the recent publication [01-02]. In the derivation of controllers in [99-22], with output and derivative information, we have used the identifiers developed in [99-02] and employed the backstepping design methodology. Under assumptions similar to those of [99-21], the controllers again guarantee the boundedness of all signals in the closed-loop system, and at the expense of increased control effort the output tracking error can be driven to an arbitrarily small neighborhood of the origin arbitrarily fast. Furthermore, the closed-loop signals satisfy a relevant disturbance attenuation inequality which, under certain conditions, implies asymptotic tracking. Similar results are obtained for the output feedback case (without derivative measurements) and presented in [01-02].

Yet another direction of generalization for the results of [99-21] is the case where in the model of the system dynamics the virtual control coefficients are also unknown-in addition to the structural uncertainty of the part of the dynamics that involve the state variables. This is addressed in [00-07]. A viable approach here is to estimate the unknown control coefficients and to incorporate these estimates into the backstepping control design, which is what we do in [00-07]. More precisely, we use the modified versions of the identifiers presented in [98-06] to estimate the unknown parameter vectors that represent the neural network approximations of the unknown nonlinearities as well as the unknown virtual control coefficients. We assume that the unknown parameters to be estimated lie in a known compact set, and use smooth projection to keep the parameter estimates within some small neighborhoods of those known compact sets. The resulting controller drives the output tracking error into a small neighborhood of the origin as quickly as desired, and keeps all other signals bounded. Moreover, a disturbance attenuation inequality with respect to an equivalent disturbance term, which is composed of the external disturbances and the approximation errors, is satisfied by the closed-loop signals. In the absence of approximation errors, this results in asymptotic tracking, provided that the external disturbances are square-integrable. In a nutshell, the results of this work constitute natural extensions of those of [98-06] and [99-21] to the more general case where the virtual control coefficients are also unknown.

\subsection{Local optimality and disturbance attenuation for nonlinear systems}

We have studied during this period also nonadaptive robust control problems with output state measurements, formulated within the framework of nonlinear $\mathrm{H}^{\infty}$ control. Here the nonlinear system is again subject to unknown disturbances in its dynamics, and the general objective is to design controllers (based on output measurements only) which would attenuate the effect of the disturbances on the output which 
has a direct feedthrough in control (which means that control is also penalized in the cost function). The solution to this problem for a general class of such systems involves the solution of a differential game defined on an infinite-dimensional state space - one generated by the cost-to-come function or the information state-which is very complex (if not impossible) to compute; see, for example, [BaBe95; JaBa95; JaBa96; HeJa99]. This challenge has prompted researchers to look into various approximation schemes, one of which is linearization of the system dynamics, resulting in controllers with local $\mathrm{H}^{\infty}$-optimality properties, without however guaranteeing any global properties. Yet another approach has been that of inverse optimal designs, which first finds a stabilizing output-feedback control law and then determines a cost functional which is minimized by that particular control law--in contrast to optimal designs which minimize cost functionals specified in advance, or equivalently solve an a priori fixed HJI (Hamilton-Jacobi-Isaacs) partial differential equation. In the case of inverse designs, first a value function is constructed using Lyapunov methods, and then an appropriate HJI equation is found, which is satisfied by that value function. Even though they guarantee stability, these inverse methods do not lead to satisfaction of a prescribed performance level. Hence there was a need to develop a new methodology that would lead to controllers that guarantee not only global stability properties but also some prescribed local performance levels. Under output measurements, this has first been accomplished in [99-05]. More specifically, we have considered in [99-05], and later in [01-03], the class of strict-feedback systems in output-feedback form where the nonlinearities depend solely on the measured output. For this class of systems, we have first constructed finite-dimensional dynamic output-feedback control laws using also the derivative of the output, which achieve local optimality and global inverse optimality with a prescribed $\mathcal{L}_{2}$-gain from the disturbances to the output. These controllers are thus locally $\mathrm{H}^{\infty}$ optimal and guarantee global stability with inverse $\mathrm{H}^{\infty}$-optimality. When only the output is available (and not its derivative), similar results are obtained, but then we have local near-optimality and semiglobal inverse optimality with a prescribed $\mathcal{L}_{2}$-gain. The recent paper [01-03] also contains an analytic example, illustrating the design procedure.

Yet another approach to overcome the difficulty associated with the infinite-dimensionality of the optimal disturbance attenuating controller in nonlinear $\mathrm{H}^{\infty}$ control is "receding-horizon" or "moving horizon," already introduced in section 2. One of the first studies that have looked at a "receding-horizon" controller design for nonlinear systems under state feedback is [MaMi90], whose results were later extended in [MiMa95] to moving-horizon observer and observer-based control design in the output feedback case. Neither study includes, however, any robustness analysis, or any connection with optimality in terms of the original problem. We have addressed this problem in [99-16] for a general class of nonlinear systems with output measurements. Our general approach involves (i) reformulation of a finite-horizon subproblem derived from the original problem, with a modified measurement equation that is consistent with the original one at the initial time of the subproblem, but is partially open loop for later times; (ii) obtaining the dissipation inequality associated with this subproblem, and deriving a controller that leads to its satisfaction; (iii) retaining the controller obtained in the earlier step as the actual control to be implemented in a pre-determined forward neighborhood of the initial time of the subproblem, advancing the time interval of the subproblem by an amount equal to that forward neighborhood, and returning to the first step for the new finite-horizon subproblem arrived at. This sequential design procedure with periodic update has been carried out in [99-16] for robust control problems formulated as minimax games where certainty equivalence [BaBe95] holds, resulting in guaranteed stability and bounded cost.

In the context of nonlinear systems subject to disturbances, another topical area we have expended 
research efforts in is investigation of the effect of high gain controllers on the stability region when the nonlinearities are not modeled in the controller design. The stability region (or region of attraction) of a dynamical system is the set of initial conditions that lead to a stable response for the system. It is a known fact that high gain in a linear feedback controller, which has various advantages when controlling linear systems with unknown disturbances (such as fast response, good disturbance rejection, and insensitivity to parameter variations), also comes with a price, which is that it may lead to vanishing stability region in the presence of neglected or unmodeled nonlinearities. The question then is whether one can come up with a design that would retain the positive features of high gain while not shrinking the stability region. We have addressed this issue in [99-08] and [01-01], where we have constructed a controller with the desired features, using $\mathrm{H}^{\infty}$ methods. High gain in the controller is achieved by letting the disturbance attenuation parameter in the linear $\mathrm{H}^{\infty}$ design approach its optimal value, while the cost on control becomes cheap. By controlling the relative rate at which these two asymptotic limits are approached, we can ensure that the stability region will not vanish, provided that we have a known bound on the unknown nonlinear disturbance. Our stability analyses in [99-08] and [01-01] are restricted to the second-order case, and use Lyapunov techniques. One interesting outcome is that the high gain creates a two time-scale behavior in the system response, thus making the closed-loop system singularly perturbed. The papers [99-08] and [01-01] also include extensive simulations to illustrate various properties of the high-gain design.

\subsection{Robust control of underactuated systems}

An underactuated system is defined to be the one where" the dimension of the space spanned by the control vector is less than the dimension of the configuration space. Simply stated, this refers to a system that has fewer control inputs than degrees of freedom. An equivalent characterization is that, as a physical (perhaps, mechanical) system, it has nonintegrable acceleration relations or dynamics. Another common description of an underactuated system is that it has second-order nonholonomic constraints--which refer to restrictions on the accelerations of the system. An example of such a system is a ship moving on a planar surface that has two forces applied at the rear of the vessel; it is underactuated because there are only two acceleration inputs while there are three degrees of freedom on the planar surface. Underactuated systems (such as underactuated vehicles) present a variety of unique control problems from both theoretical and practical perspectives. Theoretically, their reduced control authority prevents the vehicles from moving in arbitrary directions, and hence special care must be exercised in planning the motion and designing controllers

to achieve the performance objectives. From a practical perspective, there are at least two advantages to designing controllers for underactuated systems. First, a fully actuated system requires more control inputs than an underactuated system, which means that there will have to be more devices to generate the necessary forces - which adds to the cost and weight of the system. Second, underactuation provides a backup control technique for a fully actuated system. If a fully actuated system is damaged, and we have an underactuated controller available, then it may be possible to recover gracefully from the failure.

Motivated by these considerations, we have initiated a new research direction that is aimed at developing robust controllers for underactuated nonlinear systems. The standard tools used for control of nonlinear systems, such as feedback linearization and integrator backstepping [KrKaKo95], do not provide complete solutions for underactuated systems, thus requiring the development of some new tools and techniques. Our first set of results along this direction have been presented in [00-04], which develops a backstepping procedure tailored to underactuated vehicles, and describes how to use this procedure to develop a control law to perform trajectory tracking. The resulting control law can correct orientation errors to track linear 
trajectories and can track arcs of circles with a fixed offset. The paper [00-04] provides detailed derivations along with simulation results to illustrate the approach, with theoretical backing provided in [00-05]. A further recent paper, [01-06], develops algorithms for motion planning for such systems.

\subsection{Hybrid systems and their control under uncertainty}

Another class of problems we have worked on during this period is the optimal control of nonlinear systems with a controlled switching structure, where the structural changes in the system dynamics are described by a finite state Markov chain process with controlled transitions. Hence, the control has two components: one of them provides input to the continuous state dynamics, and the other one influences the transitions from one structure to another. With integral cost, defined on finite or infinite time horizons, the value functions for such stochastic control problems satisfy linearly coupled partial differential equations of the Hamilton-Jacobi-Bellman (HJB) type - as many of them as the number of states of the Markov chain. The existence and uniqueness of solutions to such partial differential equations are not guaranteed a priori, and had to be established using some generalized notions of a solution to coupled partial differential equations. In [99-18], we have settled this question by first introducing an appropriately modified and extended definition of a viscosity solution, and then establishing the existence and uniqueness of such solutions to the coupled HJB equations in both finite and infinite horizons, with the cost function having a discount factor in the latter case. We have further obtained in this paper explicit structures for the optimal controllers.

Publication [99-13] addresses another aspect of switching systems, that of high dimensionality of the Markov chain that governs the switches. Here the controlled piecewise deterministic dynamics are linear, but are perturbed by an unknown disturbance, and both the control and the disturbance are assumed to be functions of the state. The transition rates of the Markov chain, on the other hand, are taken to be control-independent, but featuring a separation between strong and weak interactions, which is modeled by a small "singular perturbation" parameter. Under a quadratic performance index, and using averaging techniques, the paper obtains low-order controllers which guarantee a precomputable level of disturbance attenuation for the original hybrid system.

Another paper on this general topic, [99-12], studies the partial measurement case for linear switching systems in the discrete time, where the control has perfect access to the continuous state but does not have access to the state of the Markov chain. The optimal solution in this case (under a quadratic performance measure) is almost impossible to obtain, which necessitates the development of techniques for obtaining reasonable approximations to the optimal controller. One such technique is provided by the receding horizon approach discussed earlier in a different context. In [99-12], this has been applied successfully to the linearquadratic hybrid control problem with partial measurements as introduced above, leading to some appealing suboptimal but stabilizing controllers.

\subsection{Risk-sensitive designs}

Recent years have witnessed intense activity on the risk-sensitive stochastic control problem, particularly for nonlinear systems, since the exponentiation of the cost brings in a parametrized degree of robustness to the underlying design, as will be discussed shortly. Our work in this area has been instrumental in this development, and we have covered over the last four years considerable ground-from addressing genuine (noncooperative) game formulations under risk sensitivity to using this framework in adaptive control.

One such new formulation and a set of new results have been reported in [97-02] and [99-01], which deal with an extension of the risk-sensitive stochastic control (RSSC) problem to differential games with multiple controllers. In particular, we consider a class of risk-sensitive stochastic nonzero-sum differential 
games with parametrized nonlinear dynamics and parametrized cost functions, with the property that if all or some of these parameters are set equal to some nominal values (in some cases zero), the differential game either becomes equivalent to a RSSC problem, or decouples into several independent RSSC problems, which in turn are equivalent to a class of stochastic zero-sum differential games. This framework has allowed us to study the sensitivity of the Nash equilibrium (NE) of the original stochastic game to changes in the values of these parameters, and to relate the $\mathrm{NE}$ (which is generally difficult to compute, and to establish existence and uniqueness for - at least directly) to solutions of RSSC problems, which are relatively easier to obtain. It has also allowed us to quantify the sensitivity of solutions to RSSC problems (and thereby to nonlinear $\mathrm{H}^{\infty}$-control problems) to unmodeled subsystem dynamics controlled by different players.

In another recent paper, [00-01], we revisit the relationship between risk-averse designs based on exponential cost functions and a class of stochastic games, which yields a robustness interpretation for risk-averse decision rules through a stochastic dissipation inequality. In other words, control laws obtained by minimizing a risk-averse cost function guarantee sensitivity bounds for the original stochastic system with respect to unmodeled plant perturbations or disturbances. This is an outcome of the equivalence between the class of risk-averse nonlinear stochastic control problems with state feedback (with a positively exponentiated integral cost) and a class of stochastic differential games with integral cost. Extensions of this equivalence to problems with noisy state measurements have been mostly elusive, with the existing results being essentially large deviation limits on the risk-sensitive control problem with "small noise," leading (in the limit) to deterministic differential/dynamic games, and hence to nonlinear $H^{\infty}$ control problems; see, for example, [JaBaE194; JaBa96]. There has also been an attempt to obtain such a relationship in risk-sensitive filtering, by again going to a deterministic limit [FlMc97], but a precise stochastic game-theoretic (and thereby robustness) interpretation for the risk-sensitive filter, particularly when the integral of the error is exponentiated, was still missing. To shed more light on this issue, we considered in [00-01] the risk-sensitive linear filtering problem, and have shown that it is equivalent (in a particular sense, delineated in the paper) to a stochastic differential game where Player 1, the minimizer, is the estimator, and Player 2, the maximizer, is the disturbance on the signal as well as the measurement process, and the players have asymmetric information. This correspondence immediately leads to a stochastic dissipation inequality for the estimation error, and thereby to a robustness interpretation of the risk-sensitive filter. In particular, it shows that risk-averse filters for linear signal-measurement models are robust (through a stochastic dissipation inequality) to unmodeled perturbations in both the signal and the measurement processes.

In an earlier work, [99-14], we had developed an integrator backstepping methodology for explicit derivation of optimum controllers for more structured RSSC problems. More specifically, for strict-feedback stochastic nonlinear systems, and under an infinite-horizon risk-sensitive cost function, we had obtained controllers that guarantee any desired positive level of long term average cost for a fixed risk-sensitivity parameter. Even though there is a limiting (large deviation) relationship between a RSSC problem and a particular nonlinear $\mathrm{H}^{\infty}$-control problem (as already alluded to), the construction of the controller presented in [99-14] for the RSSC problem differed substantially from that of the latter, presented in [98-06]. As a special case, the solution to the risk neutral control problem had also been obtained in [99-14], by simply taking the limit as the risk-sensitivity parameter approaches zero. Another special case treated was when the vector fields for the disturbance vanish at the origin of the system; in this case, the control design actually guaranteed a zero long term average cost, and the closed-loop system became asymptotically stable in the large. This work, however, did not involve cost of control. If one includes in the performance index an additional term that penalizes the control effort, then the nonlinear RSSC problem becomes substan- 
tially more difficult, even under perfect state measurements, since it involves the solution of a nonlinear Hamilton-Jacobi-Bellman equation. We have addressed this problem in [99-20] and [00-03], for stochastic strict-feedback systems described as in [99-14], but with the intensity of the Wiener process not depending on the state; furthermore, we have relaxed the optimality criterion to that of inverse optimality, where the incremental running cost is determined as part of the design process, subject to the restriction (constraint) that the controller obtained solves locally a related linear-exponential-quadratic-Gaussian (LEQG) RSSC problem where the linear dynamics are obtained as the linear approximation of the original nonlinear dynamics and the quadratic cost is specified a priori, and it is a posteriori the quadratic approximation of the running cost for the original RSSC problem. Said differently, the controller proposed, and obtained explicitly in [00-03], is locally optimal and globally inverse optimal. We also show that the controller leads to closed-loop trajectories that are bounded in probability.

Another recent work under this general topic has involved a stochastic adaptive control problem. Specifically, we consider in [00-06] an extension of the strict-feedback system model of [99-14], where now the system dynamics contain an additional linearly parameterized uncertainty, while the criterion is still the risk-sensitive cost function. To obtain a regulating risk-sensitive optimal controller for this problem, we first introduce a new class of identifiers, which are stochastic counterparts of those presented in [DiPaBa95]. These are employed to estimate the unknown parameters based on the relationship between risk-sensitive stochastic designs and stochastic games. We then use the estimates generated by these identifiers to design an adaptive controller that maintains a zero average risk sensitive cost. The controller design methodology is based on the back-stepping methodology introduced for stochastic problems in [99-14]. The derived adaptive controller also keeps the closed-loop signals bounded in probability and makes the output asymptotically approach to origin. The risk-sensitive control problem has also been consided under output measurements, in [00-10]. The designed output-feedback controller leads to zero average risk sensitive cost, boundedness in probability of all closed-loop signals, and asymptotic output regulation.

Yet another recent work, [01-05], has dealt, again in a risk-sensitive framework, with the design of decentralized controllers for interconnected systems. The class of systems considered are stochastic strictfeedback systems which interact through their outputs, and the unknown nonlinear interconnection terms are assumed to be bounded by some known functions of the outputs of the subsystems, multiplied by some unknown parameters. The controllers designed for each subsystem have access only to the information available on the respective subsystem, and they achieve an arbitrarily small value for the risk-sensitive cost for the overall system. Under this completely decentralized control scheme, all closed-loop signals remain bounded in probability.

\subsection{Hamilton-Jacobi-Isaacs equation, viscosity solutions, and infinite-dimensional systems}

As mentioned earlier, for control systems subject to unknown disturbances in their dynamics, a powerful design tool involves formulation of the problem as a differential game with two players (controller as minimizer and disturbance as maximizer) and solving the associated Hamilton-Jacobi-Isaacs (HJI) partial differential equation that the value function of the differential game satisfies. In linear-quadratic problems (that is, when the state dynamics are linear, and the cost function is quadratic in both the state and the control), this HJI equation admits (whenever it exists) an off-line computable solution, and hence the worst-case (optimally disturbance attenuating) controller can be obtained in closed form. This appealing structure of the optimal controller does not, however, generally extend to nonlinear systems, or even to linear systems with nonquadratic cost functions, as in these cases the HJI equation may not even admit a continuously 
differentiable solution. This has led to the introduction of generalized solution concepts for such equations, among which the most useful and relevant one is the viscosity solution, or even the weaker viscosity superand sub-solutions [CrEvLi84; El87; Is84]. In our work reported in [97-03], [98-03] and [99-03], we have conducted detailed studies of the viscosity (sub and super) solutions of these equations, as they arise in robust controller design (or, equivalently in our context, nonlinear $H^{\infty}$-optimal control problems), from the points of view of their existence, uniqueness, and characterization. We now discuss below the contributions of these papers in some detail.

If a nonlinear $\mathrm{H}^{\infty}$-optimal control problem is defined on an infinite-time horizon, its associated HJI equation generally admits nonunique, and in fact infinitely many, viscosity solutions. This makes it difficult to pick the relevant viscosity solution for the problem at hand, particularly when it is computed numerically. For the finite-horizon version of the problem, however, there is generally a unique viscosity solution (under appropriate conditions), which brings up the question of obtaining the viscosity solution relevant to the infinite-horizon problem as the limit of the unique solution of the finite-horizon one. In [98-03], we have addressed this question for nonlinear systems that are affine in the control and the disturbance, and under a cost function that is continuous in the state and quadratic in the control, where the control is not restricted to lie in a compact set. We have shown that the relevant viscosity solution of the HJI equation associated with the infinite-horizon control problem can be obtained as the limit of the unique viscosity solution of the HJI equation associated with a particular finite-horizon version, as the time interval goes to infinity. We have further shown that the resulting unique $\mathrm{H}^{\infty}$ controller (constructed from this viscosity solution) makes the closed-loop system globally asymptotically stable under worst-case disturbances. This result also extends to more general nonlinear systems, as long as the underlying differential game admits a saddle-point solution.

In [97-03] and [99-03], we have considered a similar class of systems, but now allowing the cost function to be discontinuous in the state as well as in the control. Such a formulation models a number of practical control problems where for example the control is required to satisfy some positivity constraints or the state is required to enter and remain in a specified region in the state space. In these problems the associated HJI equations in the finite-horizon and infinite-horizon cases do not generally admit continuous viscosity solutions, but we have been able to establish the existence of viscosity supersolutions, under the assumption that the value function is finite. We have introduced the notion of an admissible $\gamma$-attenuation state feedback controller, and have provided a method for constructing such a controller in terms of the viscosity supersolution; this construction is guaranteed to lead to an admissible controller when the value function is convex in the state. We have also established the global asymptotic stability of the closed-loop system under the $\mathrm{H}^{\infty}$ controller and the worst-case disturbance.

More recently, we have also done considerable work on robust control of infinite-dimensional systems. When the system to be controlled is infinite-dimensional (such as one whose dynamics are described by a partial differential equation or a delay-differential equation), the optimum disturbance-attenuating (worst-case) controller is infinite dimensional even when the dynamics are linear and the cost function is quadratic. A problem of theoretical and practical importance involving such systems is whether there is a finite-dimensional approximation to such a controller, with the property that it would not lead to much degradation in performance. We have addressed this problem (which has been open for quite some time) in [99-15] and [99-19], and have obtained conclusive results for time-invariant linear-quadratic problems with imperfect state measurements under a Hilbert space formulation. The approach adopted uses a Galerkin-type approximation, without any requirement for the system operator to have a complete set of eigenvectors. We have shown that if there exists an infinite-dimensional compensator satisfying a particular robustness prop- 
erty, then a finite-dimensional compensator exists and achieves the same level of robustness. We have also provided a procedure for constructing finite-dimensional compensators based on approximate solutions of two separate infinite-dimensional optimization problems, namely the worst-case regulator and worst-case observer design problems. These approximations have the property that the resulting sequence of finitedimensional controllers converge to the infinite-dimensional controller as their order increases. The order of the finite-dimensional controller depends on how much error the system can tolerate, which is measured by the difference between the desired level of disturbance attenuation and the optimum level of disturbance attenuation for the system, with the latter defined in a similar way as in the finite-dimensional case.

Another class of problems we have studied involves robust control of linear hyperbolic (wave-like) systems with additive unknown distributed disturbances, where control acts on the boundary and has access to only sampled values of the state. We have formulated this problem in [98-01] as a finite-horizon $\mathrm{H}^{\infty}$-optimal control problem, which in turn is equivalent to a zero-sum differential game. The controller is the minimizing player in this differential game, and the disturbance is the maximizing player. We have shown that a tight bound on the optimum achievable performance for this class of hyperbolic systems can be obtained in terms of the existence of a solution to a family of generalized Riccati evolution equations. A sampled-data controller that achieves any desired level of disturbance attenuation that exceeds this bound is linear, and is given in terms of the solution of another generalized Riccati evolution equation. This second evolution equation is associated with closed-loop behavior of the hyperbolic system, whereas the first family of Riccati evolution equations are associated with its open-loop behavior over each sampling interval. The control is allowed to be time-varying over each sampling interval, and hence in a sense the robust controller constructed leads to optimal waveforms over each sampling interval, as a function of the value of the state at the most recent sampling time. A numerical example included in [98-01] illustrates the theoretical results.

Yet a third class of problems we have addressed in the context of infinite-dimensional systems is the design of fixed-order finite-dimensional compensators for linear dissipative systems with additive unknown disturbances, when the criterion used is again $\mathrm{H}^{\infty}$ optimality. The design is over an infinite horizon, and the compensators utilize output measurements which are also disturbance corrupted. We have solved this problem in [98-05], for the special class of dissipative systems where the system operator (which is the infinitesimal generator of a strongly continuous contraction semigroup on the state space) has only a finite number of eigenvalues with zero real parts, which covers a large class of distributed parameter systems such as certain wave equations and beam equations. The fact that a contraction semigroup whose infinitesimal generator is a linear dissipative operator on a Hilbert space can be decomposed into a unitary part and a completely nonunitary part has enabled us to carry out the controller design on the finite-dimensional unitary subspace, where we can achieve stability and a prescribed level of disturbance attenuation using a fixed-order finite-dimensional compensator. This compensator is characterized in terms of the solutions of two algebraic Riccati equations (of dimensions equal to the order of the compensator) under a spectral radius condition. This study complements our other work [99-15] on this topic discussed earlier, where again a finite-dimensional compensator was sought for an infinite-dimensional system, but this time via a Galerkintype approximation for an infinite-dimensional compensator. In both cases, there was no requirement for the system operator to have a complete set of eigenvectors.

\subsection{Applications in compressor systems}

Another research activity carried out during this period, and particularly last year, involved an application area - the analysis and control of compressor models. One of the main challenges in the design and 
operation of compression systems is in the handling of the instabilities that arise in the unsteady fluid and structural dynamics. This is because when a turbo-machine, such as a jet engine, operates near its optimal operating point, the flow can become unstable. Such instability phenomena can lead to an undesirable reduction in the performance of compressor systems or even damage of engine components during operations.

The way a compressor system operates can be described roughly as follows: As air flow goes through the compressor, it is compressed by alternating rings of rotating blades and stationary blades; then, the mixture of fuel and compressed air is ignited, resulting in combustion that provides the output power. Two dynamic instabilities, rotating stall and surge, occur in the flow through the compressor. Rotating stall, which corresponds to a traveling wave of gas around the annulus of the compressor, occurs when a nonaxisymmetric flow disturbance develops (around the annulus of the rotor) and causes severe reduction in the performance of the compressor. As rotating stall develops, axisymmetric oscillations across the compressor system, known as surge, occur. Surge is a low-frequency, large-amplitude oscillation of the mean flow rate in the compressor, which induces high blade, causing stress levels and possible reverse flow which affects flow conditions throughout the entire compressor system. These instability phenomena must be avoided during the operation of a compressor.

Moore and Greitzer [MoGr86] developed in 1986 a relatively simple model that captures (and describes) the behavior of a compressor system. This model (known as the viscous Moore-Greitzer model) consists of a partial differential equation (PDE), which describes the behavior of disturbances in the inlet region of compressor systems, and two ordinary differential equations (ODEs), which describe the coupling of the disturbances with the mean flow. Considerable research has been carried out on the analysis and control of the stall and surge, which used simplified models obtained by a Galerkin projection of the PDE describing the stall dynamics onto its first Fourier models [KPPK95; McC90; WaMu98], but until recently little had been done on the full PDE model, which has been one of our focus areas during the last two years. Results on our study of the full PDE model have been presented in the papers [99-09], [99-17], and [00-09]. In [99-09], we considered the problem of feedback stabilization of rotating stall of a full compressor model with general cubic characteristics. We designed a distributed nonlinear controller which uses only information on the average of the disturbance velocity potential and the pressure rise coefficient (both of which are accessible), and is successful in eliminating rotating stall as the pressure reaches its maximum. In the papers [99-17] and [00-09], we have established the existence of a local center manifold for the Moore-Greitzer PDE model with viscous term, as the throttle coefficient is reduced to a certain value. This is accomplished by converting the PDE into an abstract evolution equation, and proving that the system operator of the linearized model is an infinitesimal generator of an analytic semigroup. We then show that the qualitative behavior of the local flow in the compressor can be determined from the flow of two appropriate scalar differential equations on this center manifold. Based on an analysis of the reduced system, we have subsequently established the important result that the Moore-Greizer PDE model with viscous term is unstable when its linearized system has a pair of purely imaginary eigenvalues.

In a fourth paper, [00-08] (see also [99-06]), we have undertaken a study of control of a multi-mode Moore-Greitzer model, which is obtained through a multi-mode expansion of the disturbance velocity using a Galerkin projection-leading to an approximation of the PDE by a finite number of ordinary differential equations. Our main approach in controlling this system was to let the throttle characteristic not touch the rotating envelope surface, so that only one nominal equilibrium point exists for the closed-loop system. In accordance with this, we have developed a nonlinear state feedback controller, depending on a setpoint parameter, which renders the closed-loop dynamic model globally asymptotically stable at a location 
prescribed by the set-point parameter. The controller thus prevents the compression system state from entering rotating stall or surge. Furthermore, the controller also converges to a stabilizing distributed controller for the full model in an appropriate space norm. Simulations included in [00-08] illustrate the theoretical results.

\section{BIBLIOGRAPHY}

[Bar94] V.Barbu, The $H^{\infty}$-problem with control constraints. SIAM J.Control and Optimization, 32: 952-964, 1994.

[Ba91] T. Başar, A dynamic games approach to controller design: Disturbance rejection in discrete time. IEEE Transactions on Automatic Control, AC-36(8): 936-952, 1991.

[BaBe95] T. Başar and P. Bernhard, $H^{\infty}$-Optimal Control and Related Minimax Design Problems: A Dynamic Game Approach, Birkhäuser, Boston, 2nd edt., 1995.

[BaHa84] T. Başar and A. Haurie, Feedback equilibria in differential games with structural and modal uncertainties. In Advances in Large Scale Systems, J.B.Cruz, Jr., edt JAI Press Inc, Connecticut, vol. 1, pp. 163-201, 1984.

[BaOl99] T. Başar and G.J. Olsder, Dynamic Noncooperative Game Theory, SIAM Series in Classics in Applied Mathematics, Philadelphia, 1999.

[BeEl96] A. Bensoussan and R.J. Elliott, General finite dimensional risk sensitive problems and small noise limits. IEEE Transactions on Automatic Control, vol. AC-41(2): 210-215, February 1996.

[BeSc85] A. Bensoussan and J.H. Van Schuppen, Optimal control of partially observable stochastic systems with an exponential-of-integral performance index. SIAM J. Control and Optimiz., 23(4): 599-613, 1985.

[CaEu92] P.Cardaliaguet and G.Euvrard, Approximation of a function and its derivative with a neural network. Neural Networks., vol. 5, pp. 207-220, 1992.

[ChSp95] D.F. Chichka and J.L. Speyer, An adaptive controller based on disturbance attenuation. IEEE Transactions on Automatic Control, vol. AC-40(7): 1220-1233, July 1995.

[CrEvLi84] M.G. Crandall, L.C. Evans and P.L. Lions, Some properties of viscosity solutions of HamiltonJacobi equations. Transactions of the American Mathematical Society, 282(2): 487-502, 1984.

[Da92] M. Davis, Markov Models and Optimization, Monographs on Statistics and Applied Probability, Chapman and Hall, London, 1992.

[DiBa97] G. Didinsky and T. Başar, Minimax adaptive control of uncertain plants. ARI, Springer Verlag, 50(1):3-20, March 1997.

[DiBaBe93] G. Didinsky, T. Başar, and P. Bernhard, Structural Properties of minimax controllers for a class of differential games arising in nonlinear control. Systems \& Control Letters , 21: 433-441, December 1993.

[DiPaBa95] G. Didinsky, Z. Pan, and T. Başar, Parameter identification for uncertain plants using $\mathrm{H}^{\infty}$ methods. Automatica, 31(9): 1227-1250, September 1995.

[DoGlKhFr89] J.C. Doyle, K. Glover, P.P. Khargonekar and B.A. Francis, State space solutions to standard $H_{2}$ and $H_{\infty}$ control problems, IEEE Transactions on Automatic Control, vol. AC-34(8): 831-847, 1989.

[El87] R.J. Elliott, Viscosity Solutions and Optimal Control, Longman Scientific and Technical, Pitman Research Notes in Mathematics Series, London, 1987.

[ElAgMo95] R.J. Elliott, L. Aggoun and J.B. Moore, Hidden Markov Models: Estimation and Control, Springer-Verlag, New York, 1995.

[FlMc92] W.H. Fleming and W.M. McEneaney, Risk sensitive optimal control and differential games. In Stochastic Theory and Adaptive Control, Lecture Notes in Control and Information Sciences, 184: 185-197, Springer-Verlag, Berlin, 1992.

[FIMc97] W.H. Fleming and W.M. McEneaney, Risk sensitive and robust nonlinear filtering. In Proc. 36th IEEE Conf. Decision and Control, San Diego, CA, 1997 
[FlSo93] W.H. Fleming and H.M. Soner, Controlled Markov Processes and Viscosity Solutions, Springer Verlag, New York, NY, 1993.

[GIDo88] K. Glover and J.C. Doyle, State-space formulae for all stabilizing controllers that satisfy the $H^{\infty}$ norm bound and relations to risk sensitivity. Systems \& Control Letters, 11: 167-172, 1988.

[GrLi95] M. Green and D.J.N. Limebeer, Linear Robust Control, Prentice Hall, New Jersey, 1995.

[HeJa99] J.W.Helton and M.R.James, 'Extending $H_{\infty}$ control to nonlinear systems, SIAM Frontiers in Applied Mathematics, 1999.

[Is84] H. Ischii, Uniqueness of unbounded viscosity solutions of Hamilton-Jacobi equation. Indiana University Mathematics Journal, 33: 721-749, 1984.

[Is93] A. Isidori, New results on nonlinear $\mathrm{H}_{\infty}$-control via measurement feedback. In Advances in Dynamic Games and Applications (T.Başar and A. Haurie, eds.), Birkhäuser, Boston, MA, 1993, pp. 56-69.

[IsAs92] A. Isidori and A. Astolfi, Disturbance attenuation and $\mathrm{H}_{\infty}$-control via measurement feedback in nonlinear systems, IEEE Transactions on Automatic Control, AC-37(9): 1283-1293, 1992.

[IsKa95] A. Isidori and W. Kang, $\mathrm{H}^{\infty}$ control via measurement feedback for general nonlinear systems. IEEE Transactions on Automatic Control, AC-40: 466-472, March 1995.

[Ja73] D.H. Jacobson, Optimal stochastic linear systems with exponential performance criteria and their relation to deterministic differential games. IEEE Transactions on Automatic Control, AC-18(2): 124-131, 1973.

[JaBa95] M.R. James and J.S. Baras, Robust $H_{\infty}$ output feedback control for nonlinear systems. IEEE Transactions on Automatic Control, vol. AC-40(6): 1007-1017, June 1995.

[JaBa96] M.R. James and J.S. Baras, Partially observed differential games, infinite-dimensional HamiltonJacobi-Isaacs equation, and nonlinear $H_{\infty}$ control. SIAM J. Control and Optimiz., 34(4): 1342-1364, July 1996.

[JaBaEl94] M.R. James, J.S. Baras, and R.J. Elliott, Risk-sensitive control and dynamic games for partially observed discrete-time nonlinear systems. IEEE Transactions on Automatic Control, vol. AC-39: 780-792, 1994.

[KPPK95] M. Krstic, J. M. Protz, J. D. Paduano, and P. V. Kokotović, Backstepping designs for jet engine stall and surge control. Proc.34th IEEE Conf. Decision and Control, pp. 3049-3055, 1995.

[KrKaKo95] M. Krstic̀, I. Kanellakopoulos, and P.V. Kokotovic̀, Nonlinear and Adaptive Controller Design, Wiley, New York, 1995.

[MaMi90] D. Q. Mayne and H. Michalska, Receding horizon control of nonlinear systems. IEEE Transactions on Automatic Control, AC-35(7): 814-824, July 1990.

[McC90] F. McCaughan. Bifurcation analysis of axial flow compressor stability. SIAM J. of Appl. Math., 50:1232-1253, 1990.

[MiMa95] H. Michalska and D. Q. Mayne, Moving horizon observers and observer-based control. IEEE Transactions on Automatic Control, AC-40(6): 995-1007, June 1995.

[MoGr86] F.K. Moore and E.M. Greitzer, A theory of post-stall transients in axial compression systems: Part I-development of equations. ASME J. Engineering for Gas Turbines and Power, 108:68-76, 1986.

[Na96] H. Nagai, Bellman equations of risk-sensitive control. SIAM J. Control and Optimization, 34(1): 74$101,1996$.

[PaBa95a] Z. Pan and T. Başar, $\mathrm{H}^{\infty}$-control of Markovian jump systems and solutions to associated piecewisedeterministic differential games. In G.J. Olsder, editor, Annals of Dynamic Games, vol. 3, pp. 61-94, Birkhäuser, 1995.

[PaBa95b] Z. Pan and T. Başar, Robustness of minimax controllers to nonlinear perturbations. J. Optimization Theory and Applications, 87(3): 631-678, December 1995.

[PaBa96] Z. Pan and T. Başar, Model simplification and optimal control of stochastic singularly perturbed systems under exponentiated quadratic cost. SIAM J. Control and Optimization, 34(5): 1734-1766, September 1996 . 
[PaSa93] J.Park and I.W.Sandberg, Approximation and radial-basis-function networks. Neural Computation, pp. 305-316, 1993.

[Po92] M.J.D.Powell, The theory of radial basis function approximation in 1990. In W.Light, editor, Advances in Numerical Analysis. vol. 2, Clarendon Press, Oxford, 1992. pp. 102-205.

[RhSp91] I. Rhee and J.L. Speyer, A game theoretic approach to finite-time disturbance attenuation problem. IEEE Trans. Automat. Contr, AC-36: 1021-1032, 1991.

[Sc91] A.J. Van der Schaft, A state space approach to nonlinear $\mathrm{H}_{\infty}$ control. Systems and Control Letters, 16: 1-8, 1991.

[Sc92] A.J. Van der Schaft, $\mathrm{L}_{2}$-gain analysis of nonlinear systems and nonlinear $\mathrm{H}_{\infty}$ control. IEEE Transactions on Automatic Control, AC-37: 770-784, 1992.

[St92] A.A. Stoorvogel, The $H_{\infty}$ Control Problem: A State Space Approach, Prentice Hall, 1992.

[UcFu89] K. Uchida and M. Fujita, On the central controller: Characterizations via differential games and LEQG control problems. Systems and Control Letters, vol. 13(1), pp. 9-13, 1989.

[WaMu98] Y. Wang and R. M. Murray, Effects of the shape of compressor characteristics on actuator requirements for rotating stall control. Proc. American Control Conference, 1998.

[Wh90] P. Whittle, Risk-Sensitive Optimal Control, John Wiley \& Sons, Inc., Somerset, New Jersey, 1990.

[Wh91] P. Whittle, A risk-sensitive maximum principle: The case of imperfect state observation, IEEE Transactions on Automatic Control, AC-36(7): 793-801, 1991.

[Za81] G. Zames, Feedback and optimal sensitivity: Model reference transformations, multiplicative seminorms, and approximate inverses. IEEE Trans. Automat. Control, AC-26: 301-320, 1981.

\section{PUBLICATIONS SUPPORTED BY THE CURRENT GRANT}

We provide in this section a list of publications which have resulted from our research activities supported by the DOE Grant, covering the four-year period (since June 1997). Contents and contributions of these publications have been discussed in Section 3 of this Final Report.

[97-01] Z. Pan and T. Başar, "Adaptive controller design and disturbance attenuation for SISO linear systems with noisy measurements," Proc. 1997 European Control Conference, Brussels, Belgium, July (1-4) 1997.

[97-02] T. Başar, "Nash Equilibria of Risk-Sensitive Nonlinear Stochastic Differential Games," Preprints, 1997 ISDG Workshop on Dynamic Games, Sils-Maria, Switzerland, August (21-22) 1997.

[97-03] M. Xiao and T. Başar, "Viscosity supersolutions of a class of Hamilton-Jacobi-Isaacs equations arising in nonlinear $\mathrm{H}^{\infty}$ control," Proc. 36th IEEE Conference on Decision and Control, San Diego, CA, pp. 1761-1766, December (10-12) 1997.

[98-01] M. Xiao and T. Başar, " $H^{\infty}$-optimal boundary control of hyperbolic systems with sampled measurements," Proc. 37th IEEE Conference on Decision and Control, Tampa, FL, pp. 2830-2835, December 16-18, 1998.

[98-02] S. Lu and T. Başar, "Robust nonlinear system identification using neural network models," IEEE Transactions on Neural Networks, 9(3):407-429, May 1998.

[98-03] M. Xiao and T. Başar, "Nonlinear $\mathrm{H}^{\infty}$ control with unbounded controls: viscosity solutions and feedback design," Proc. IFAC Nonlinear Control Symposium, Enschede, Netherlands, July 1-3, 1998.

[98-04] T. Vallée and T. Başar, "Incentive Stackelberg solutions and the genetic algorithm," Preprints of the International Symposium on Dynamic Games and Applications, Maastricht, the Netherlands, pp. 633639, July 6-8, 1998.

[98-05] M. Xiao and T. Başar, "Fixed-order finite-dimensional compensators for $H^{\infty}$ optimal control of dissipative systems in infinite dimensions," Preprints of the International Symposium on Dynamic Games and Applications, Maastricht, the Netherlands, pp. 680-685, July 6-8, 1998. 
[98-06] Z. Pan and T. Başar, "Adaptive controller design for tracking and disturbance attenuation in parametricstrict-feedback nonlinear systems," IEEE Transactions on Automatic Control, AC-43(8):1066-1083, August 1998.

[99-01] T. Başar, "Nash equilibria of risk-sensitive nonlinear stochastic differential games," J. Optimization Theory and Applications, 100(3):479-498, March 1999.

[99-02] I.E. Tezcan and T. Başar, "Disturbance attenuating adaptive controllers for parametric strict feedback nonlinear systems with output measurements," ASME J. Dynamic Systems, Measurement and Control, 121:48-57, March 1999.

[99-03] M. Xiao and T. Başar, "Nonlinear $\mathrm{H}^{\infty}$ controller design via viscosity supersolutions of the Isaacs equation," In W. McEneaney, G.G. Yin, Q. Zhang, editors, Stochastic Analysis, Control, Optimization and Applications, Birkhäuser, pp. 151-170, 1999.

[99-04] T. Vallée and T. Başar, "Off-line computation of Stackelberg solutions with the genetic algorithm," J. Computational Economics, 13(3):201-209, June 1999.

[99-05] K. Ezal, A.R. Teel, P.V. Kokotović, and T. Başar, "Disturbance attenuating output-feedback control of nonlinear systems with local optimality," Proc. 1999 American Control Conference, San Diego, CA, pp. 2578-2583, June 2-4, 1999.

[99-06] M. Xiao and T. Başar, "Analysis and control of multi-mode Moore-Greitzer compressor models," Proc. 1999 American Control Conference, San Diego, CA, pp. 2647-2651, June 2-4, 1999.

[99-07] G. Arslan and T. Başar, "Robust identification for tracking in systems with unknown nonlinearities," Proc. 1999 American Control Conference, San Diego, CA, pp. 1057-1061, June 2-4, 1999.

[99-08] G.J. Toussaint and T. Başar, "Achieving nonvanishing stability regions with high-gain cheap control using $\mathrm{H}^{\infty}$ techniques: The second-order case," Proc. 1999 American Control Conference, San Diego, CA, pp. 1279-1283, June 2-4, 1999.

[99-09] M. Xiao and T. Başar, "Rotating stall control of MG3 compressor models governed by partial differential equations," Proc. 14th IFAC Congress, vol. E, pp. 183-188, Beijing, P.R. China, July 5-9, 1999.

[99-10] T. Başar and T. Vallée, "Computation of the closed-loop Stackelberg solution using the genetic algorithm," Proc. 14th IFAC Congress, vol. M, pp. 69-74, Beijing, P.R. China, July 5-9, 1999.

[99-11] T. Vallee, Ch. Deissenberg, and T. Başar, "Optimal open loop cheating in dynamic reversed linearquadratic Stackelberg games," Annals of Operations Research, 88:217-232, 1999.

[99-12] J.B.R. do Val and T. Başar, "Receding horizon control of discrete-time Markov jump linear systems," J. Economic Dynamics and Control, 23(8):1099-1131, August 1999.

[99-13] Z. Pan and T. Başar, " $\mathrm{H}^{\infty}$ control of large scale jump linear systems via averaging and aggregation," International $J$. of Control, 72(10):866-881, 1999.

[99-14] Z. Pan and T. Başar, "Backstepping controller design for nonlinear stochastic systems under a risksensitive cost criterion," SIAM J. Control and Optimization, 37(3):957-995, 1999.

[99-15] M. Xiao and T. Başar, "Finite-dimensional compensators for the $\mathrm{H}^{\infty}$-optimal control of infinite dimensional systems via a Galerkin-type approximation. SIAM J. Control and Optimization, 37(5):1614-1647, 1999.

[99-16] R. Blauwkamp and T. Başar, "A receding-horizon approach to robust output feedback control for nonlinear systems. 38th IEEE Conference on Decision and Control, Phoenix, AZ, pp. 4879-4884, Dec 7-10, 1999.

[99-17] M. Xiao and T. Başar, "Center manifold of the viscous Moore-Greitzer PDE model. 38th IEEE Conference on Decision and Control, Phoenix, AZ, pp. 668-673, Dec 7-10, 1999.

[99-18] M. Xiao and T. Başar, "Optimal control of piecewise deterministic nonlinear systems with controlled transitions: Viscosity solutions, their existence and uniqueness. 38th IEEE Conference on Decision and Control, Phoenix, AZ, pp. 4712-4717, Dec 7-10, 1999.

[99-19] M. Xiao and T. Başar, "Finite-dimensional compensators for the $\mathrm{H}^{\infty}$-optimal control of infinite dimensional systems via a Galerkin-type approximation. 38th IEEE Conference on Decision and Control, Phoenix, AZ, pp. 1095-1100, Dec 7-10, 1999. 
[99-20] T. Başar and C. Tang, "Locally optimal risk-sensitive controllers for nonlinear strict-feedback systems," 38th IEEE Conference on Decision and Control, Phoenix, AZ, pp. 115-120, Dec 7-10, 1999.

[99-21] G. Arslan and T. Başar, "Robust output tracking for strict-feedback systems using neural-net based approximators for nonlinearities. 38th IEEE Conference on Decision and Control, Phoenix, AZ, pp. 29872992, Dec 7-10, 1999.

[99-22] G. Arslan and T. Başar, "Robust output-feedback control of strict-feedback systems with unknown nonlinearities. 38th IEEE Conference on Decision and Control, Phoenix, AZ, pp. 4748-4753, Dec 7-10, 1999.

[00-01] T. Başar, "Risk-averse designs: From exponential cost to stochastic games," in T.E. Djaferis and J.C. Schick, editors, System Theory: Modeling, Analysis and Control, Kluwer, 2000, pp. 131-144.

[00-02] M. Xiao and T. Başar, " $\mathrm{H}^{\infty}$ control of a class of infinite-dimensional linear systems with nonlinear outputs," in V. Gaitsgory and J. Filar, editors, Annals of Dynamic Games, vol. 5, pp. 47-80, Birkhäuser, 2000.

[00-03] T. Başar and C. Tang, "Locally optimal risk-sensitive controllers for nonlinear strict-feedback systems," J. Optimization Theory and Applications, 105(3):521-541, June 2000.

[00-04] G.J. Toussaint, T. Başar and F. Bullo, "Tracking for nonlinear underactuated surface vessels with generalized forces," Proc. IEEE International Conference on Control Applications, pp. 355-360, September 25-27, 2000, Anchorage, Alaska.

[00-05] G. Toussaint, T. Başar and F. Bullo, " $\mathrm{H}^{\infty}$-optimal tracking control techniques for nonlinear underactuated systems," Proc. 39th IEEE Conf. Decision and Control, pp. 2078-2083, December 12-15, 2000, Sydney, Australia.

[00-06] G. Arslan and T. Başar, "Adaptive control of stochastic strict-feedback systems under a risk-sensitive criterion," Proc. 12th IFAC Symposium on System Identification, Santa Barbara, CA, June 21-23, 2000.

[00-07] G. Arslan and T. Başar, "Robust output tracking for uncertain strict-feedback systems with unknown virtual control coefficients," Proc. 12th IFAC Symposium on System Identification, Santa Barbara, CA, June 21-23, 2000.

[00-08] M. Xiao and T. Başar, "Analysis and control of multi-mode axial flow compression system models," ASME J. Dynamic Systems, Measurement and Control, 122(3):393-401, September 2000.

[00-09] M. Xiao and T. Başar, "Center manifold of the viscous Moore-Greitzer PDE model," SIAM J. Applied Mathematics, 61(3):855-869, 2001.

[00-10] G. Arslan and T. Başar, "Output-feedback control of stochastic strict-feedback systems under an exponential cost criterion," Proc. 39th IEEE Conf. Decision and Control, pp. 2450-2455, December 12-15, 2000, Sydney, Australia.

[01-01] G. Toussaint and T. Başar. Achieving nonvanishing stability regions with high-gain cheap control using $\mathrm{H}^{\infty}$ techniques: The second order case. Systems \& Control Letters, 44:79-89, September 2001.

[01-02] G. Arslan and T. Başar. Disturbance attenuating controller design for strict-feedback systems with structurally unknown dynamics. Automatica, 37(8):1175-1188, August 2001.

[01-03] K. Ezal, A.R. Teel, P.V. Kokotovic, and T. Başar. Disturbance attenuating output-feedback control of nonlinear systems with local optimality. Automatica, 37(6):805-818, June 2001.

[01-04] C. Tang and T. Başar, "Stochastic stability of singularly perturbed nonlinear systems," Proc. 40th IEEE Conf. Decision and Control, Orlando, Florida, December 4-7, 2001 (to appear)

[01-05] G. Arslan and T. Başar, "Decentralized risk-sensitive controller design for strict-feedback systems," Proc. 40th IEEE Conf. Decision and Control, Orlando, Florida, December 4-7, 2001 (to appear)

[01-06] G. Toussaint, T. Başar, and F. Bullo, "Motion planning for nonlinear underactuated vehicles using $\mathrm{H}^{\infty}$ techniques," Proc. 2001 American Control Conf., pp. 4097-4102, June 25-27, 2001, Arlington, Virginia.

[02-01] T. Başar, "Paradigms for robustness in controller and filter designs," J. Macroeconomic Dynamics, 2002 (to appear). 